\title{
Children with a Specific Phobia do better in Individual CBT than Group CBT and guided parent-led CBT
}

\author{
By Dr Anna McKinnon
}

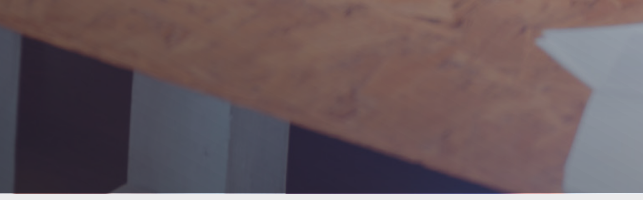

Bio: Anna McKinnon is a registered clinical psychologist based at the Centre for Emotional Health Clinic, Macquarie University. My research is broadly focussed on investigating the cognitive, behavioural, emotional and biological factors maintaining psychological disorders in the aftermath of trauma.

Children often present to health care settings with highly impairing and disabling anxiety disorders, including Specific Phobia, Social Anxiety Disorder, Generalised Anxiety Disorder and Separation Anxiety Disorder.

Cognitive Behaviour Therapy (CBT) is a psychological treatment focussed on assisting children and families develop healthy ways of coping with the thoughts, feelings, and behaviours maintaining anxiety disorders. It is an active, structured, and time limited therapy, suggested as the first-choice treatment for children with anxiety disorders due to its widespread support in clinical trials. Around $60 \%$ of children that take part in trials of CBT will improve substantially, no longer having a diagnosis at the end of treatment.

In attempting to access services, parents of children with anxiety and/or other health professionals involved, are typically eager to select a treatment which will give their child the best chance possible of making improvements. CBT programs are now available in a variety of formats (e.g., length, number of sessions etc.), giving parents and children more choice than ever. Importantly, some families can now decide whether their child will participate in a CBT program delivered in a group setting at a clinic, guided parent-led format at home, or individual face to face treatment at a clinic. Some parents may have little option but to enrol their child in a guided parent-led program for many reasons, including living a long distance from a clinic, demanding work schedules, or the fact that many children simply refuse to attend a clinic for sessions.

But, which CBT treatment format - individual, group or guided parent-led CBT - offers the child the best chance of improving their symptoms? And does the nature of the child's anxiety diagnosis make a difference to their chances of improving in the different treatment formats? For families trying to decide between different programs for their child, there is currently no clear guidance on this issue.

Individual, group and guided parent-led CBT each have their pros and cons. Individual treatment is expensive, but the therapist can personalise the therapy to directly meet the child's needs. Group programs can be prescriptive and lack flexibility. Despite this potential limitation, they are less expensive to run and it can be less stigmatising and more supportive for the child to learn the skills alongside his/her peers. There are also logical reasons why certain categories of anxiety problems might be suited to different treatment formats. For example, socially anxious children might find participating in group CBT to be too overwhelming. On the flipside, this format could be more beneficial as 
the child might have invaluable opportunities to practice social exposure skills (e.g., starting conversations) in the group. Whilst there have been some randomised controlled trials evaluating whether group or individual CBT is more beneficial for child anxiety, these studies have not had large enough samples to definitely test whether the child's anxiety diagnosis makes a difference to outcomes. Furthermore, these studies have typically used brief self-report questionnaires (i.e. pen-and-paper format) to measure the child's psychiatric problems, leading to inconclusive findings.

In our study (published in the Journal of Child Psychology and Psychiatry), we pooled data from multiple previously published RCTs carried out in Australia, United Kingdom, Norway, Denmark Germany, Switzerland, USA, and the Netherlands. We compared outcomes of individual, group and guided-parent led CBT for children with a primary anxiety disorder (i.e., the child's most impairing anxiety disorder) of Specific Phobia, Social Anxiety Disorder, Generalised Anxiety Disorder or Separation Anxiety Disorder. The sample included 1253 anxious children (5 to 18 years) that had received a course of CBT for child anxiety. This unique sample was the largest available to date to explore this important research question.

At each site, a trained diagnostician interviewed children and their parents using a structured clinical interview to determine the nature of any anxiety disorders experienced by the child. For children with more than one diagnosis, the clinician also determined the disorder having the biggest negative impact on the child. Interviews also occurred immediately after treatment and at longer term follow-up.

We utilised a statistical approach involving multi-level data modelling, using a within-subjects level regression modelling approach accounting for differences observed across sites. We found that anxious children with Generalised Anxiety Disorder, Social Anxiety Disorder and Separation Anxiety Disorder benefitted substantially from taking part in CBT, doing equivalently well in individual, guided parent-led and group CBT. Similarly, children with Specific Phobias also benefitted from all three CBT treatment formats, but they differed from other children in that they did better in individual CBT than either group or guided parent-led CBT.

Our findings offer several important implications for guidance and policy. When parents are accessing treatment, it may be appropriate to explain to them that their child will have equivalent chances of responding across all three formats, meaning parents can place more weight on pragmatic considerations when selecting between treatment formats (e.g., ability of parents to bring child to appointments, demand for service, waiting lists etc.). From a public policy perspective, this finding supports the idea that cheaper lower intensity group and guided parent led CBT formats could be offered to families before more expensive approaches are offered.

Correspondingly, when parents of children with Specific Phobia's are trying to decide on a treatment it may be appropriate to tell them that enrolment in an individual program will give the child the best chance of improving. We speculate that this may be because face to face formats are critical for treating the substantial avoidance behaviours associated with phobias. The individual format allows the psychologist to tailor protocols by providing specific psychoeducation surrounding the fear (e.g., education about dog safety) as well as utilising in-session guided exposure to feared situations, and training parents to be efficacious in guiding their child through the exposure process.

Our findings add to a growing body of literature investigating why it is that some children with anxiety disorders respond better to CBT programs than others. However, our approach of pooling data from RCTs and utilising multilevel modelling offers a less controlled examination of these questions than is found in an RCT. Therefore, future RCTs looking at these questions and including health economics are needed before definitively answering this question.

Studies such as this, which explore factors influencing how well a child responds to treatment, are imperative as they add to our understanding of the optimal conditions for delivering treatment to anxious children.

Key point

- Children presenting for treatment of a primary Social Phobia in clinical settings should routinely be offered individual CBT before group and guided parent-led CBT.

- The allocation of children with Generalised Anxiety Disorder, Social Anxiety Disorder and Separation Anxiety Disorder to individual, group and guided parent-led CBT could incorporate other considerations (e.g., pragmatic considerations).

- For children with a primary Generalised Anxiety Disorder, Separation Anxiety Disorder, and Social Anxiety Disorder, lower intensity group and guided parent led CBT formats could be offered to families before more expensive approaches are offered.

- Future RCTs assessing the effect of individual, group and guided parent-led CBT on levels of anxiety as well as the cost-effectiveness of these approaches.

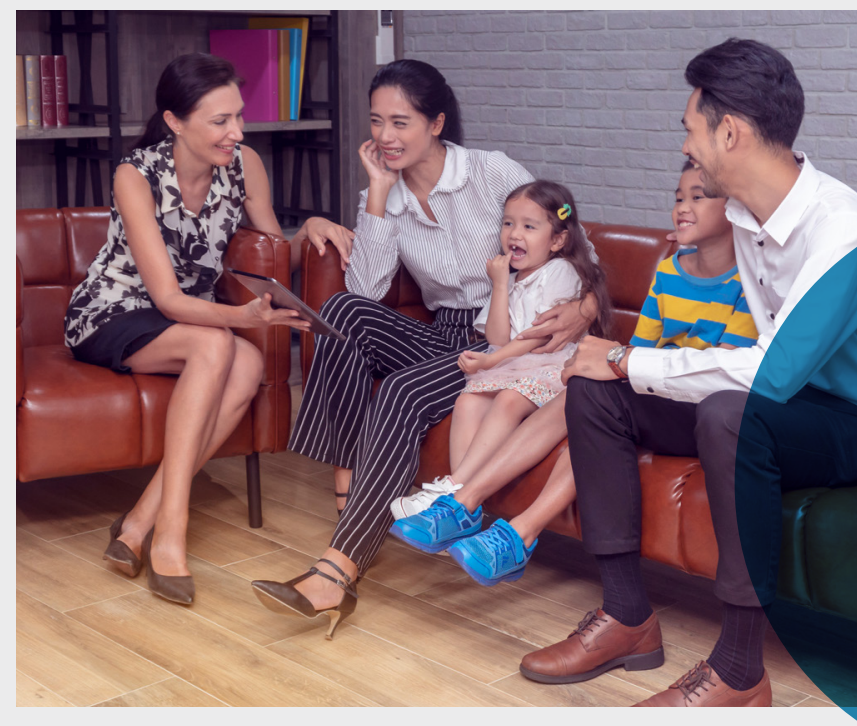

